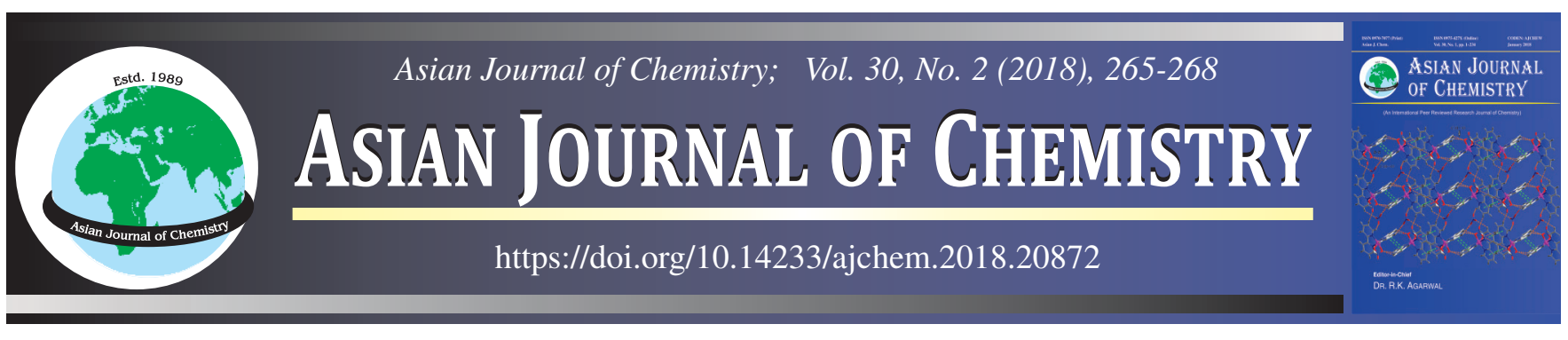

\title{
Surface Tension of Mixed Solutions of Methyl Acetate and Ethyl Acetate with Cyclohexanone and Acetophenone
}

\author{
LeSEDi RATSATSi and JAMIL AhMAD*
}

Chemistry Department, University of Botswana, P/Bag 00704, Gaborone, Botswana

*Corresponding author: E-mail: ahmadj@mopipi.ub.bw

Received: 17 June 2017;

Accepted: 22 September 2017;

Published online: 31 December 2017;

AJC-18689

\begin{abstract}
Results of measurement of surface tension on mixed solutions of methyl acetate and ethyl acetate with cyclohexanone and acetophenone are reported. The values are interpreted in terms of intermolecular interactions. Surface tension deviations from linear, additive values, show that the acetophenone interacts with either methyl or ethyl acetate less strongly as compared to cyclohexanone. Similarly ethyl acetate interacts less strongly with either acetophenone or cyclohexanone compared to methyl acetate. The results are interpreted in terms of the relative bulk and the polarizability of the interacting molecules.
\end{abstract}

Keywords: Surface tension deviations, Molecular interactions, Methyl acetate, Ethyl acetate, Cyclohexanone, Acetophenone.

\section{INTRODUCTION}

Measurement of surface tension of liquid mixtures can yield useful information about molecular interactions between components. Surface tension of mixtures plays an important role in many industrial processes, such as spreading, energy transfer at an interface, liquid-liquid extraction and froth floatation [1-3]. It is a conveniently and rapidly measurable property and is accurate. Industries using knowledge gained from surface tension of mixtures include those dealing with detergents, paints, mineral extraction, agrochemicals and petroleum products.

This article describes the results of measurement of surface tension on mixtures of each of methyl acetate and ethyl acetate with cyclohexanone and acetophenone. These systems were chosen because they allow the effect of the size of the alkyl group (methyl versus ethyl) on deviation from linear behaviour to be studied and also the effect of the neighboring groups to the keto group (as in cyclohexanone and acetophenone) can be observed.

\section{EXPERIMENTAL}

The chemicals 'used were purified by redistillation, unless their purity was over $99 \%$ as per their manufacturer's specifications.

Various solutions were prepared by dissolving appropriate masses of each component in hexane and making up the volume in volumetric flasks. The solutions were transferred to stoppered bottles, which were then placed in thermostat baths at the desired temperatures. The temperatures were controlled to within $\pm 0.1 \mathrm{~K}$.

Surface tension was measured with a Kruss K9 tensiometer using the platinum ring detachment method. The ring used was model R21 with a diameter of $(19.0 \pm 0.5) \mathrm{mm}$. Prior to use, the ring was first rinsed with distilled water and then heated to red-hot in a Bunsen burner flame to burn off any impurities. A $50 \mathrm{~mL}$ sample vessel was filled to about twothird capacity with the liquid being investigated. The instrument was checked by measuring the surface tension of distilled water at $25^{\circ} \mathrm{C}$, which was found to be close to the literature value for pure water, $71.99 \mathrm{mN} / \mathrm{m} \mathrm{[4].} \mathrm{All} \mathrm{the} \mathrm{measurements}$ were repeated two to three times with the ring rinsed and heated to red-hot between measurements and the container rinsed with the next sample before taking the reading. To ascertain the limits within which the temperature of the sample varied during the course of the measurement, the temperature of the sample was taken before and after the surface tension measurement. The limit within which the temperature varied was $\pm 0.2 \mathrm{~K}$.

\section{RESULTS AND DISCUSSION}

Surface tension: Surface tension - composition plots for all the four systems are shown in Figs. 1-4.

If the interactions between molecules of the components are relatively weak, the surface tension of the mixture is expected to deviate from linearity [5-7]. It is energetically more favourable for the component with the lower surface tension to be preferentially expelled from the bulk to the surface. If the 


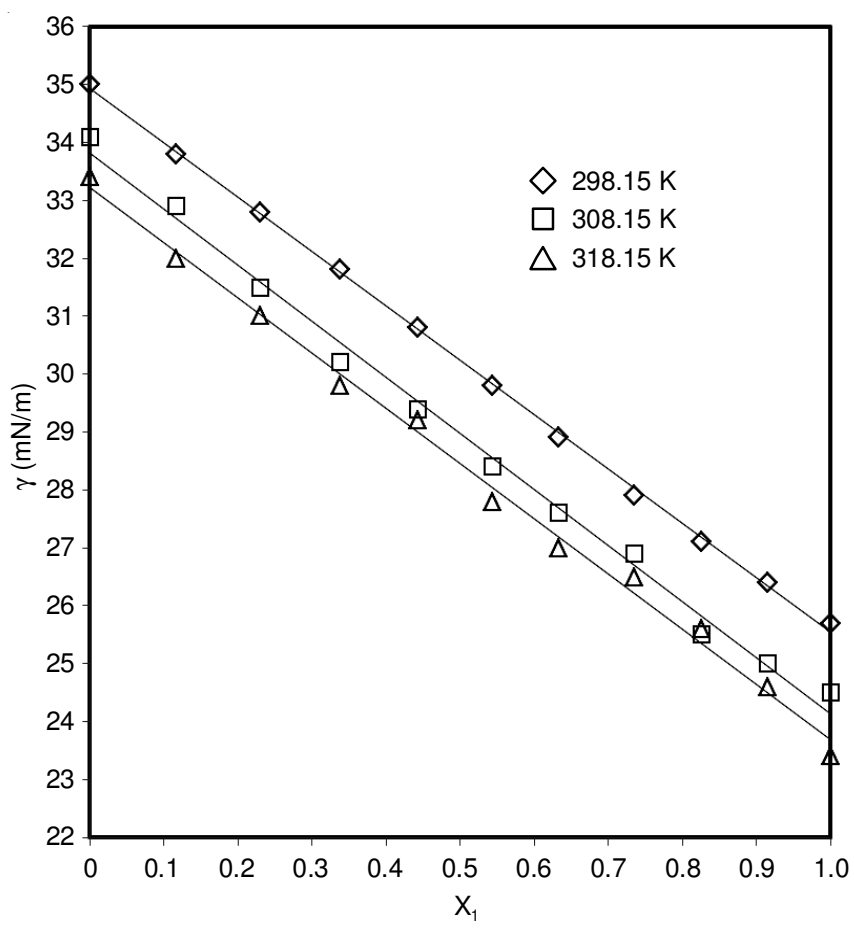

Fig. 1. Surface tension against mole fraction for the methyl acetate (1) and cyclohexanone (2) mixtures at different temperatures

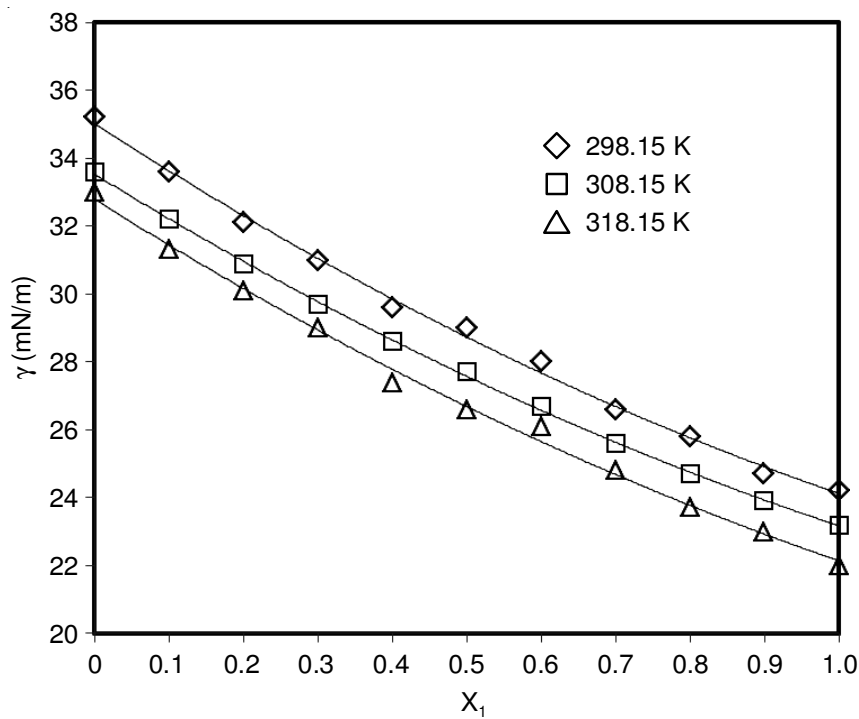

Fig. 2. Surface tension against mole fraction for the ethyl acetate (1) and cyclohexanone (2) mixtures at different temperatures

intermolecular interaction is relatively weak, this is achieved readily and there results a negative deviation from linearity. On the other hand the molecular interactions between the components are relatively strong, the preferential expulsion of molecules of either of the components is not likely and the composition of the surface reflects the composition in the bulk and the resultant surface tension varies linearly with composition.

Of the four systems studied, only one (methyl acetatecyclohexanone) shows a linear behaviour (Fig. 1), with all other systems showing a negative deviation. Thus cyclohexanoneethyl acetate mixture shows a negative deviation in the surfacetension composition curves, while cyclohexanone-methyl

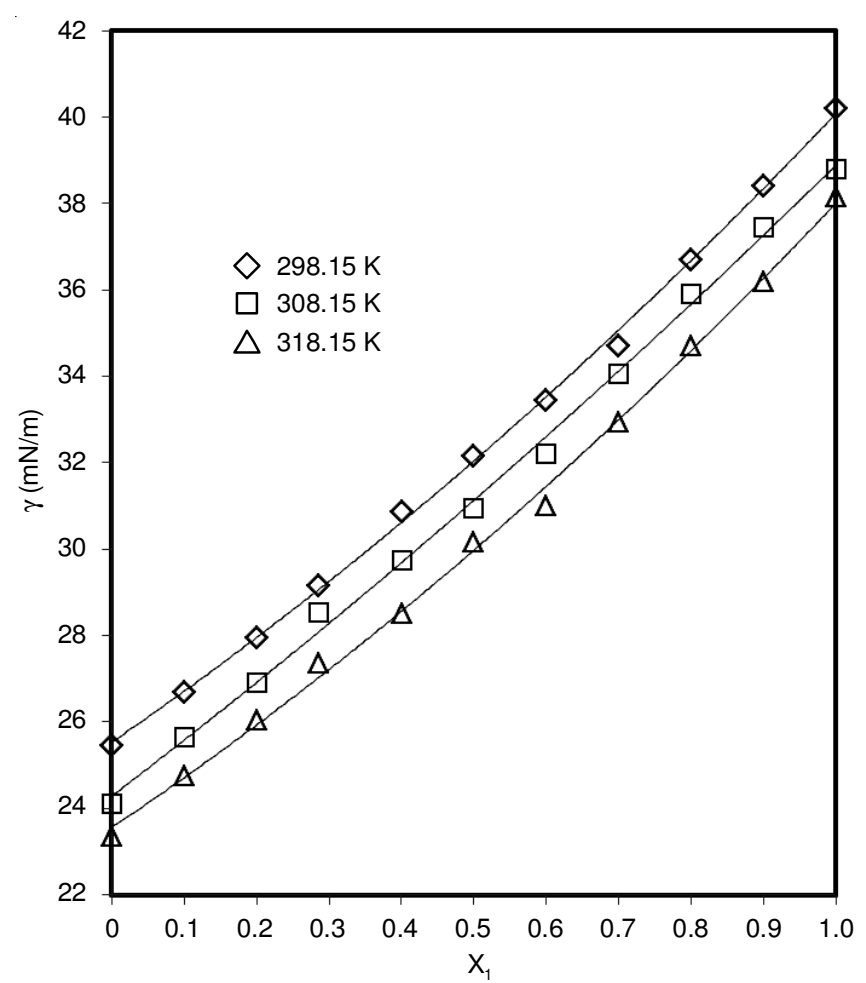

Fig. 3. Surface tension against mole fraction for the acetophenone (1) and methyl acetate (2) mixtures at different temperatures

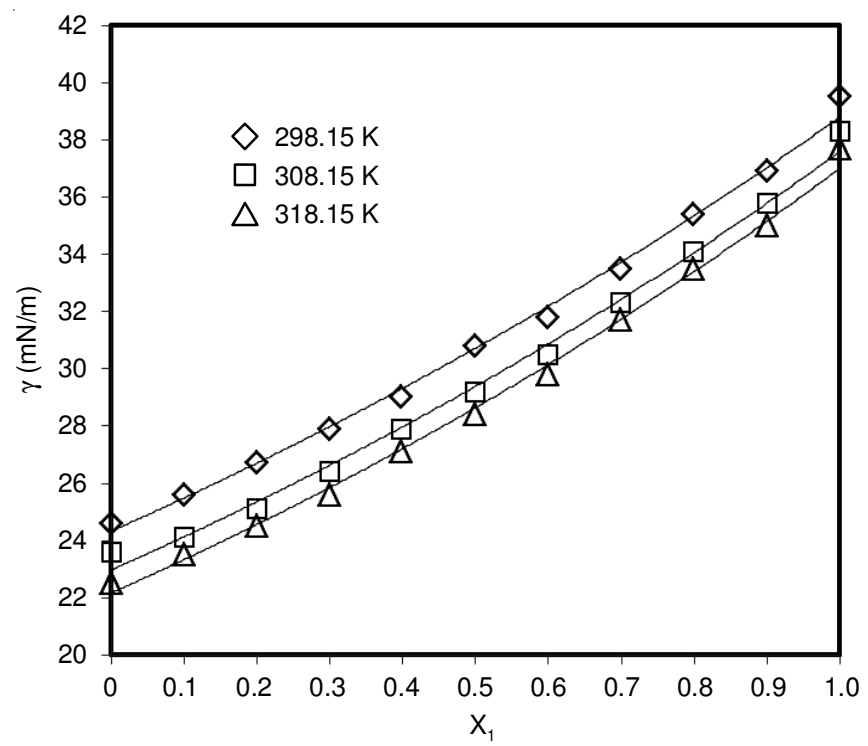

Fig. 4. Surface tension against mole fraction for the acetophenone (1) and ethyl acetate (2) mixtures at different temperatures

acetate mixture shows a linear behaviour. This difference can be explained by the different extent of intermolecular interaction between the molecules of the systems studied. From the curves, one can conclude that cyclohexanone's interactions with ethyl acetate are weaker than they are with methyl acetate. This difference in interactions can be understood in terms of the ethyl group being more massive and having lower polarizability compared to the methyl group, resulting in weaker dipole-dipole interactions.

Comparing methyl acetate-cyclohexanone system with methyl acetate-acetophenone, one finds a similar difference 
in trend. The former shows a linear behaviour, while the latter has a negative deviation from linearity. A comparison between the structures of acetophenone and cyclohexanone reveals that the dipole-dipole interaction of methyl acetate with cyclohexanone with its exposed carbonyl group will be stronger than with the bulkier acetophenone with its relatively sheltered $\mathrm{CO}$ moiety. Hence the linear behaviour of methyl acetate-cyclohexanone system as opposed to negative deviation for the methyl acetate-acetophenone system.

In the same vein one should expect the acetophenoneethyl acetate to show a negative deviation. Fig. 4 shows the confirmation in this view.

Surface tension deviations: In view of the foregoing statement, it is apparent that surface tension deviation from the ideal, additive, value can give an insight into the relative intermolecular interactions between the components. Surface tension deviation $(\Delta \gamma)$ can be defined as:

$$
\Delta \gamma=\gamma-\left(\mathrm{X}_{1} \gamma_{1}+\mathrm{X}_{2} \gamma_{2}\right)
$$

Here $\gamma$ is the measured surface tension of the solution of component 1 and $2, \mathrm{X}_{1}$ and $\mathrm{X}_{2}$ are their mole fractions in the solution and $\gamma_{1}$ and $\gamma_{2}$ are the respective surface tensions of the pure liquids. Plots of $\Delta \gamma$ against composition of the solution are given in the Figs. 5-8. The data points are fitted to a polynomial of degree three.

It is apparent from an examination of these graphs that the deviation from ideality is greater for acetophenone-ethyl acetate system compared to acetophenone-methyl acetate, implying weaker intermolecular interaction in the former com-

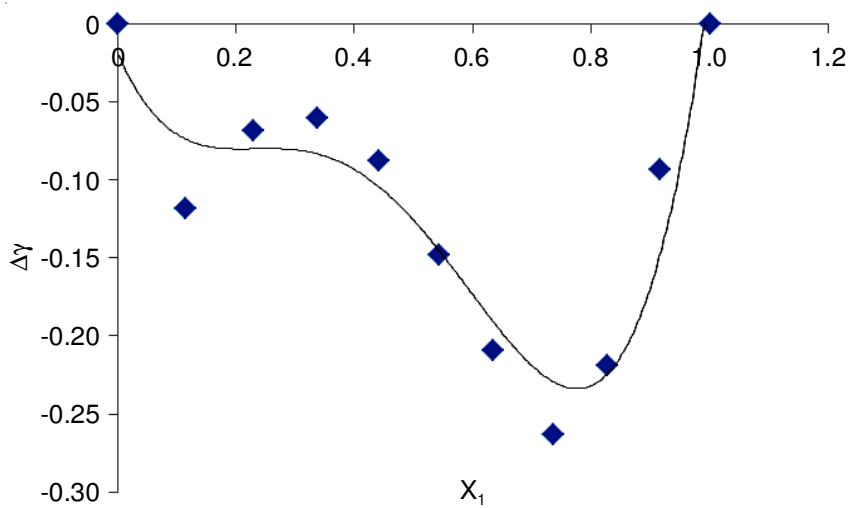

Fig. 5. Surface tension deviation, $\Delta \gamma(\mathrm{mN} / \mathrm{m})$, against mole fraction for methyl acetate (1) and cyclohexanone (2) mixtures

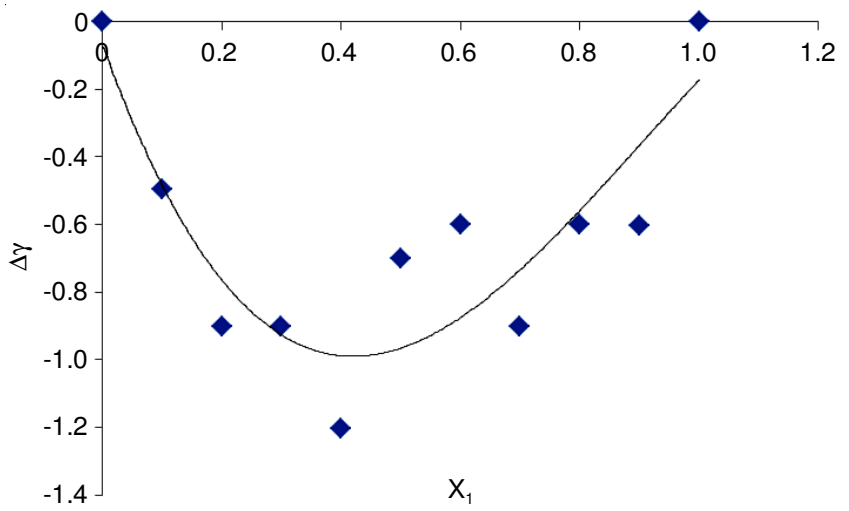

Fig. 6. Surface tension deviation, $\Delta \gamma(\mathrm{mN} / \mathrm{m})$, against mole fraction for ethyl acetate (1) and cyclohexanone (2) mixtures

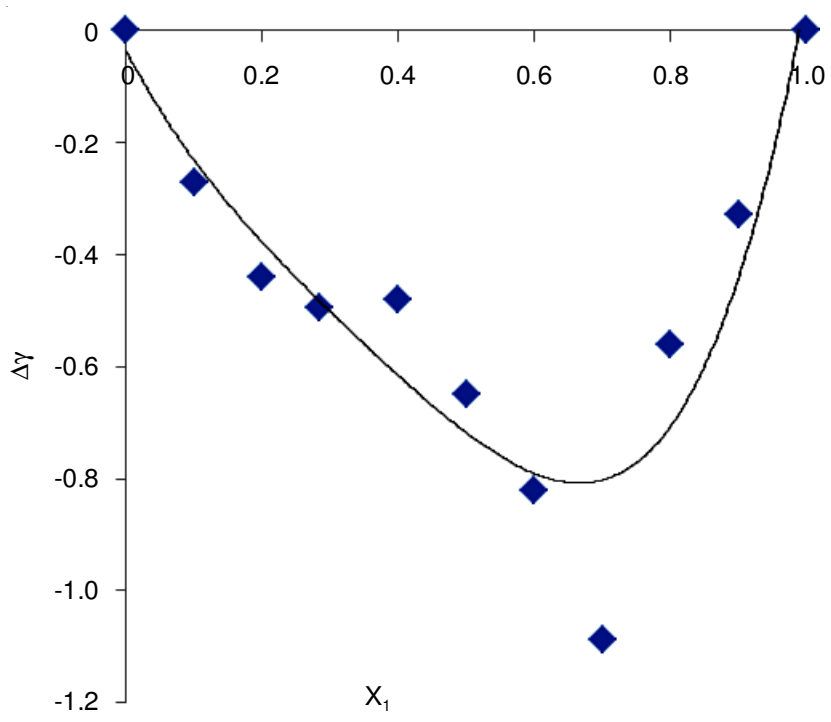

Fig. 7. Surface tension deviation, $\Delta \gamma(\mathrm{mN} / \mathrm{m})$, against mole fraction for acetophenone (1) and methyl acetate (2) mixtures

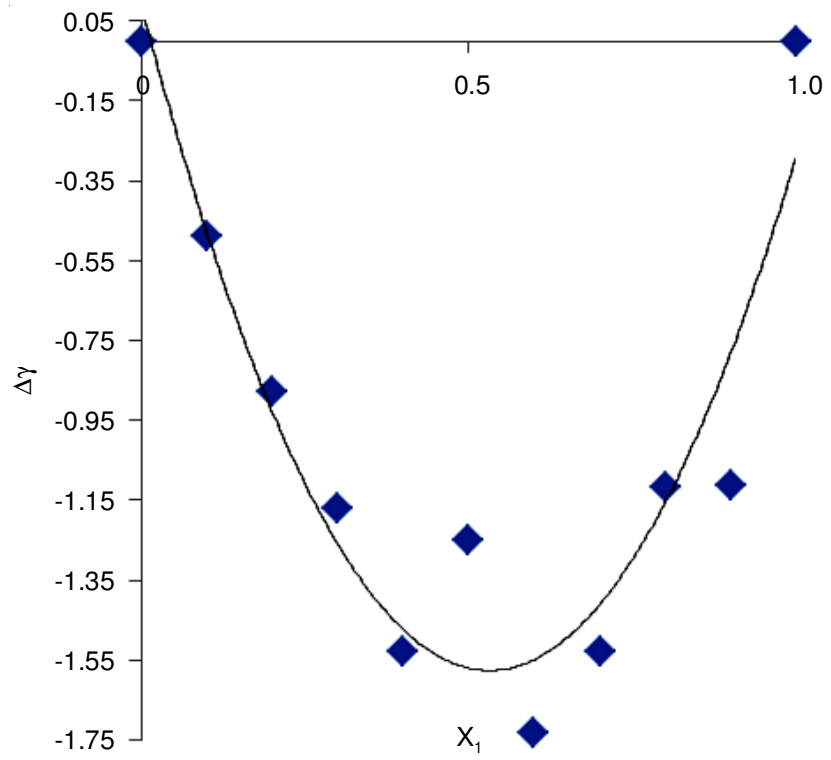

Fig. 8. Surface tension deviation against mole fraction, $\Delta \gamma(\mathrm{mN} / \mathrm{m})$, for acetophenone (1) and ethyl acetate (2) mixtures

pared to the latter system. Similarly based on the value of $\Delta \gamma$ it is concluded that acetophenonone interacts less strongly with either ethyl acetate or methyl acetate compared to cyclohexanone. Moreover ethyl acetate interacts with either acetophenone or cyclohexanone less strongly as compared to methyl acetate.

The relative weakness of the interaction of acetophenone with either ethyl acetate or methyl acetate compared to that of cyclohexanone can be understood in terms of the relative bulk of the two molecules, with the $\mathrm{CO}$ group of acetophenone being relatively less exposed as compared to that of cyclohexanone, resulting in weaker interactions with either ethyl acetate or methyl acetate.

\section{Conclusion}

Surface tension of mixed systems can give an insight into the intermolecular interactions between the components. Surface tension deviations from linear, additive, behaviour 
show that acetophenone interacts with either ethyl acetate or methyl acetate less strongly as compared to cyclohexanone. Similarly ethyl acetate interacts with either acetophenone or cyclohexanone less strongly than methyl acetate does.

\section{REFERENCES}

1. A.A. Rafati and E. Ghasemian, J. Colloid Interface Sci., 328, 385 (2008);

https://doi.org/10.1016/j.jcis.2008.09.014.

2. A. Bagheri and A.H. Amiri-Majed, J. Chem. Thermodyn., 51, 45 (2012); https://doi.org/10.1016/j.jct.2012.02.017.
3. L.F. Ramírez-Verduzco, A. Romero-Martínez and A. Trejo, Fluid Phase Equilib., 246, 119 (2006);

https://doi.org/10.1016/i.fluid.2006.05.026

4. N.B. Vargaftik, B.N. Volkov and R.D. Voljak, J. Phys. Chem. Ref. Data, 12, 817 (1983); https://doi.org/10.1063/1.555688.

5. S. Azizian and N. Bashavard, Colloids Surf. A Physicochem. Eng. Asp., 240, 69 (2004);

https://doi.org/10.1016/j.colsurfa.2004.03.011.

6. R.K. Shukla, N. Awasthi, V.S. Gangwar, S.K. Singh and K. Srivastava, Res. J. Recent Sci., 1, 224 (2012).

7. R.C. Reid, J.M. Prausnitz and B.E. Poling, The Properties of Gases and Liquids, McGraw Hill, pp. 646 (1987). 